\title{
Intrinsic Piezo-Nanogenerator Integrated Flexible Self-Charging Supercapacitor Power Cell: Overview and Outlook
}

\author{
Karamjyoti Panigrahi $* \$$ \\ * School of Materials science \& Nanotechnology, Jadavpur University, Jadavpur, Kolkata-700032, India \\ ${ }^{\$}$ Current address: Indian Institute of Science Education and Research (IISER), Dr. Homi Bhabha Road, Pune, \\ India, 411008 \\ E-Mail: karamjyoti.panigrahi@acads.iiserpune.ac.in
}

https://doi.org/10.54162/SD01-25201/04

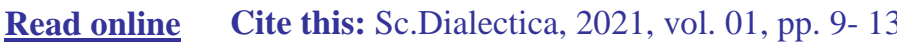

Keywords: Supercapacitor, Nanogenerator, Piezoelectric

\begin{abstract}
Coupling of energy harvesting unit with the energy storage one has already gained considerable attention in the development of self-powered portable gadgets. Nanogenerators (NGs) and flexible supercapacitors (SCs), both are considered as leading energy devices in their respective domains. Integration with each other opens up the new possibility of self-charging supercapacitors. Among, the NGs piezo-electric NGs are preferred over triboelectric NGs for integration with SC to avoid additional circuit complexity. Here, device architecture, the working principle, and imperative parameters regarding piezo-electric NG-based selfpowered SCs are sequentially discussed. Finally, a conclusion is drawn from some recent works, and remarks are provided for cultivating its overall performance.
\end{abstract}

\section{Introduction}

To address serious threats of global warming and scarcity of fossil fuels, scientists and engineers are engaged in intense research efforts for the design and fabrication of energyefficient devices to exploit sustainable and clean energy [1]. As energy harvesting and storage are two completely different techniques, their coupling with each other paves the pathway towards next-generation self-powered energy devices. An investigation on a fusion of solar cell/nanogenerator (NG) or fuel cell with either batteries or SCs via intrinsic and/or extrinsic hybrid systems has grabbed attention among various research groups. Intrinsic integration is preferred over extrinsic as no additional power management system/circuitry is required for the former one. Thus, an intrinsically fused energy device in a single architecture is capable of energy harvesting, converting, and storing [2]. Among several conversion methods, conversion of low-frequency mechanical energy to useful voltage output via piezoelectric and triboelectrification $a k a \mathrm{NG}$ has become a prime choice due to low maintenance cost, easy fabrication, and durability [3]. Alternatively, high power density, prolonged lifetime compared to batteries, fast-charging-discharging rate makes electrochemical capacitors (ECs) or supercapacitors (SCs) as next-generation energy-storage devices [4]. Thus, integration of SCs and NGs in a single device offers the next-generation hybrid self-powered supercapacitor. Besides, the rapid growth of device miniaturization with flexible and portable aspects opens up the development of flexible all-solid-state supercapacitors by using gel electrolyte in between the positive and negative terminal of a flexible substrate. The use of gel electrolyte here plays the dual role of electrolyte and separator and solves two inherent glitches (i) electrolyte leakage and (ii) short-circuit between two electrodes.

In this short review, device architecture $\&$ mechanism of intrinsic self-powered SCs based on NG is focused with an emphasis on the influential design parameters to provide a brief outline and future perspectives.

\section{Device Architecture}

In general, for all flexible solid-state SC, two configurations are largely followed termed as "symmetric" and "asymmetric" configuration. Conventional symmetric SCs consist of electrodes (cathode and anode) with the same type of electrode materials (same mass loading, same charge storage mechanism) which suffers from "narrow potential window" limitation cramping the energy density value of overall symmetric SC device [5]. Nevertheless, a similar charge storage phenomenon (at both electrodes) removes device design complexity and improves the overall stability of the device. On the other hand, in case of asymmetric configuration integration of two dissimilar materials having different/same charge storage phenomenon, different mass loading overcomes the 
"narrow potential window limitation" and improves the overall energy density of it. Dissimilar durability of cathode and anode materials in asymmetric configuration greatly hinders the performance of the device. Thus, proper design along with a choice of materials are prerequisite requirements for efficient asymmetric SC device design [6]. Transition metal oxides, sulfides, and carbon-based materials i.e. reduced graphene oxide ( $\mathrm{rGO}$ ), graphene, and recently 2-D carbides termed as MXenes, are mostly explored as electrode materials [4]. To obtain the desired flexibility, conducting substrate e.g. carbon cloth/fabric (CF), graphene foam, carbon paper, Ag or Au deposited cotton fabrics and papers, Al foils, PET gain considerable attention [7]. Polymer electrolytes with conducting proton $(\mathrm{H}+)$ or $\mathrm{Li}+$ or $\mathrm{OH}$ - and ionic electrolytes with ionic species are mostly preferred to avoid potential window limitation of aqueous electrolytes. The salt to polymer ratio mainly controls the properties and physical attributes of the polymer electrolyte. When the polymer content is higher compared to the salt the polymer acts as a matrix, but in the case of high salt content, the polymer acts as a binder to provide the conduction network for ion transport [8]. The number of available ions largely depends on the hydration rate of those polymer electrolytes.

The energy harvesting unit (piezo/tribo NG) can be integrated into two ways with the energy storage unit based upon their working mechanism (i) direct/intrinsic: composed mainly of piezoelectric NG and (ii) indirect/extrinsic (with rectifier): composed of mainly triboelectric NG. In the first one, the nanogenerator is directly integrated with the SC as the separator in between the two electrodes of SC. On the other hand, in the case of indirect integration methods instead of direct integration of nanogenerator within the SC, a bridge rectifier circuit is coupled with the output of nanogenerator with the input of SC. However, usage of a bridge-rectifier circuit possesses a gridlock in the indirect methods as some of the harvested energy is consumed by the bridge- rectifier; hence for utilizing fully harvested energy, rectification circuit-free SCs are preferred.

\section{Mechanism}

The mechanism of the rectifier-free self-charged supercapacitor can be discussed based upon the piezo electrochemical process (as depicted in Figure 1 with 5 consecutive stages). At the beginning state (Stage 1), when any type of mechanical deformation is initiated, the electrochemical equilibrium in between two electrodes and electrolyte is preserved because of no electrochemical reaction in between them. This state is termed as "discharge state" or "equilibrium state". Now when compressive stress is applied, the internal equilibrium of the piezo/tribo separator film is disturbed and polarization takes place within it, as depicted in stage 2 . Thus, the developed piezo- potential across the piezo separator drives electrolytic ions towards the positive and negative electrodes (Stage 3) via a combination of (i) physical ion adsorption kinetics and (ii) movement through ion-conducting pathways through the electrolyte gel [2]. Without using any type of external source input, the movement of electrolyte ions towards the electrodes initiates a potential difference across the two electrodes which is termed as "self-charging". During the self-charging, the potential of the two electrodes increases in the opposite direction up to a certain operating voltage until equilibrium is achieved. The piezoelectric field induced ions migration towards the respective electrode in the electrolyte screens the generated piezo-potential across the separator. Consequently, the ionic movement-induced electrochemical imbalance is countered by the redox (oxidation at positive terminal and reduction at negative terminal) or faradic reactions at the surface of the electrode as shown in step 3. This leads to the accumulation of free electrons at the positive terminal and vice versa at the negative terminal. The liberated free electrons at the positive terminal can migrate towards the opposite electrode via any two paths, through the inside of the piezoelectric driven SC or the current collector. An external circuit consisting of an electrochemical monitoring system or measuring the voltage via multimeter before and after compression provides the idea of an actual electron flow path. When the compressive stress is removed completely (Step 4), the piezoelectric potential disappears along with the generated piezopotential, a small portion of electrolyte ions transfers back to the electrolyte for compensation. Additionally, the reverse back of electrons towards the positive electrode also stops. This leads to no electrochemical reaction at both of the electrodes, and the device returns to its previous state. In this way, the harvested mechanical energy from continuous compression and relaxation is directly stored in the SC without the use of any rectification circuits. From the mechanism, it is evident that the output voltage depends upon the generated piezoelectric field as well as the faradic or redox reaction of the electrode material. Based on the above working principle, various research groups have designed intrinsic self-powered SCs mainly using PVDF or PVDF along with other nanofiller as the piezoelectric separator [1,9$11]$.

Nevertheless, some research groups are focused on using some natural products as piezo-separator in between the two electrodes [12].

\section{Influential Parameters}

The performance of the piezoelectric-based self-powered SC is determined by how quickly the SCs get charged up to a certain potential under compressive stress (normally palm tapping) of specific value. The obtained potential is 


\section{Compressive stress}

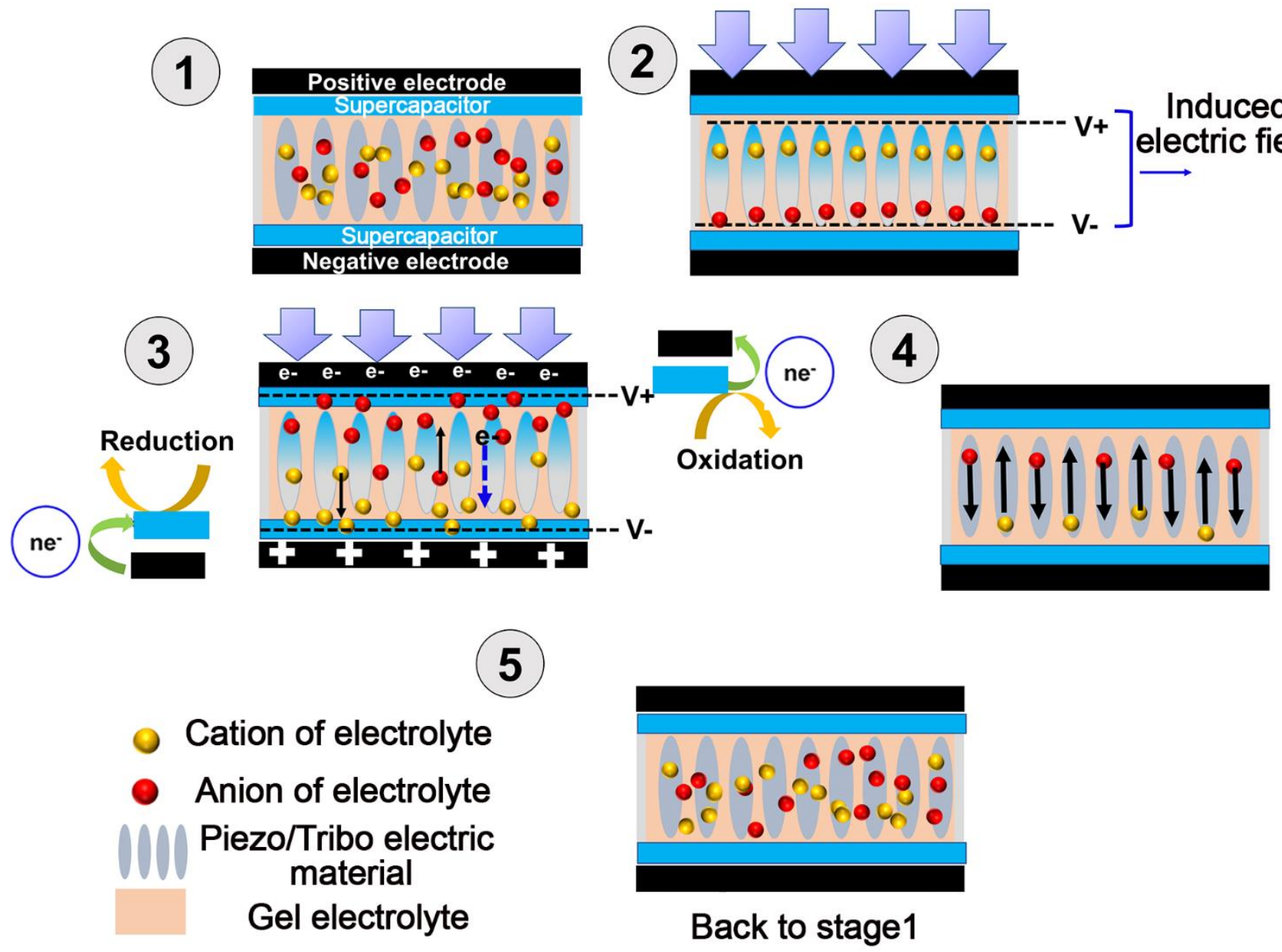

Figure 1: Operation principle of self-powered supercapacitor with piezo-separator under mechanical stress.

also increased with the frequency enhancement as well as with the compressive stress increment. The output voltage follows a step-like nature with the time when continuous tapping and de-tapping are periodically applied. However, precaution is necessary as excessive compressive stress or frequency can deform the overall device structure. Apart from voltage, the high current, as well as the slow discharging time and long cycle stability, are prerequisites for SC. Also, the choice of electrolyte will be based upon the used piezo-separator as the electrolyte should remain non-reactive towards the piezo-material throughout the whole operation period. On the other hand, the porosity of the piezoelectric NG film which works as the separator is also a topic of concern, because a high porous separator boosts the hassle-free ion migration from one electrode to another. Most commonly used piezoelectric membranes are made up of polymers e.g. PVDF, P(VDF-TrFE), etc. which suffer from the less porous network as well as stretchability issue [13]. Piezoelectric semiconductor materials are embedded in the piezoelectric separator to improve the porosity as well as piezo-potential across the piezoseparator [8]. To overcome the stretchability issue, Zhou et. al came up with a new concept by introducing a stretchable piezo electrolyte made up of piezoelectric material potassium sodium niobate (KNN) mixed within conventional gel electrolyte $\mathrm{PVA} / \mathrm{KOH}$ and directly used as separator and energy harvesting unit [13]. This idea thus opens up a new possibility for the development of selfpowered SC using piezoelectric-electrolyte.

\section{Conclusion and Outlook}

Hybrid self-powered supercapacitors are emerging as a promising device for the development of next-generation flexible SCs. This will solve the external charging issue of an SC without any additional electronic circuit. However, a comprehensive understanding of the entire piezoelectrochemical process is still in the developing stage, as the whole ion transfer (from electrolytes to electrodes 
through piezo-separator) due to harvested piezo-potential within the piezo-electric separator is based upon theoretical validation. Recent advances in piezo-electrochemical spectroscopy (electrochemical station along with piezoelectric field measuring unit) will further strengthen the understanding regarding the working principle. However, there is ample scope of development in the coming days, as the obtained output voltage from the hybrid device is not very much high compared to extrinsic powered SCs. The voltage maximization can be accomplished by improving parameters i.e. ionic mobility through a separator, the redox or faradic reaction rate at the electrodes, and generated piezo-potential in the piezo matrix. The ionic mobility of the electrolyte can be improved by improving the porous network of the piezo-separator. Moreover, the ionic electrolyte can be used instead of water-based gel electrolyte, but special attention should be provided to its interaction with the piezo-electric matrix. The redox or faradic reaction rate can be boosted by improving the charge storage ability of the electrode materials. In that case, metalorganic frameworks (MOFs) and carbon frameworks (COFs) have great potential, as they already obtained special attention in SC development. For practical applications of intrinsic self-powered SCs, its long-time operation needs improvement which is a challenging issue for this type of hybrid device. Of course, the greatest challenge is the enhancement of the output voltage from a single hybrid device as their output in maximum cases is limited in millivolt to volt range. Improved research efforts on optimization, usages of sophisticated measuring techniques combined with further development of device architecture, will unlock the present gridlocks of these intrinsic self-powered SCs, subsequently paving the way towards a new kind of flexible Power Cell for the future energy industry.

\section{References}

[1] A. Ramadoss, B. Saravanakumar, S. W. Lee, Y. S. Kim, S. J. Kim, Z. L. Wang, " Piezoelectric-driven self-charging supercapacitor power cell", ACS Nano, 2015, vol. 9, pp. 4337-4445.

[2] K. Krishnamoorthy, P. Pazhamalai, V. K. Mariappan, S. S. Nardekar, S. Sahoo and S.J. Kim, "Probing the energy conversion process in piezoelectric-driven electrochemical self-charging supercapacitor power cell using piezoelectrochemical spectroscopy", Nat. Commun., 2020, vol. 11, pp. 1-11.

[3] S. Paria, R. Bera, S.K. Karan, A. Maitra, A.K. Das, S.K. Si, L. Halder, A. Bera, and B.B. Khatua, "Insight into Cigarette Wrapper and Electroactive Polymer Based Efficient TENG as Biomechanical Energy Harvester for
Smart Electronic Applications", ACS Appl. Energy Mater., 2018, vol. 1, pp. 4963-4975.

[4] S. Najib and E. Erdem, "Current progress achieved in novel materials for supercapacitor electrodes: mini review", Nanoscale Adv., 2019, vol. 1, pp. 2817-2827.

[5] W.G. Pell, B.E. Conway, "Peculiarities and Requirements of Asymmetric Capacitor Devices Based on Combination of Capacitor and Battery-Type Electrodes", $J$. Power Sources, 2004, vol. 136, pp. 334 - 345.

[6] Y. Shao, M.F. El-Kady, J. Sun, Y. Li, Q. Zhang, M. Zhu, H. Wang, B. Dunn \& R.B. Kaner, "Design and mechanisms of asymmetric supercapacitors", Chemical reviews, 2018 vol. 118, pp. 9233-9280.

[7] Y. Yang, "A mini-review: emerging all-solid-state energy storage electrode materials for flexible devices", Nanoscale, 2020, vol. 12, pp. 3560-3573.

[8] S. Alipoori, S. Mazinani, S.H. Aboutalebi, and F. Sharif, "Review of PVA-based gel polymer electrolytes in flexible solid-state supercapacitors: Opportunities and challenges", Energy Storage, 2020, vol. 27, pp. 101072.

[9] A. Rasheed, W. He, Y. Qian, H. Park, and D.J. Kang, "Flexible Supercapacitor-Type Rectifier-free Self-Charging Power Unit Based on a Multifunctional Polyvinylidene Fluoride-ZnO-rGO Piezoelectric Matrix", ACS Appl. Mater. Interfaces, 2020, vol. 12, pp. 20891-20900.

[10] P. Pazhamalai, K. Krishnamoorthy, V.K. Mariappan, S. Sahoo, S. Manoharan, and S.J. Kim, “A High Efficacy SelfCharging $\mathrm{MoSe}_{2}$ Solid-State Supercapacitor Using Electrospun Nanofibrous Piezoelectric Separator with Ionogel Electrolyte," Adv. Mater. Interfaces, 2018, vol. 5, pp. 1800055-1800064.

[11] Y. Lu, Y. Jiang, Z. Lou, R. Shi, D. Chen, and G. Shen, "Wearable supercapacitor self-charged by P (VDF-TrFE) piezoelectric separator", Prog. Nat. Sci., 2020, vol. 12, pp. 174-179.

[12] A. Maitra, S.K. Karan, S. Paria, A.K. Das, R. Bera, L. Halder, S.K. Si, A. Bera, and B.B. Khatua, "Fast charging self-powered wearable and flexible asymmetric supercapacitor power cell with fish swim bladder as an efficient natural bio-piezoelectric separator", Nano Energy, 2017, vol. 40, pp. 633-645.

[13] D. Zhou, N. Wang, T. Yang, L. Wang, X. Cao, and Z. Wang, "Piezoelectric Nanogenerator Promotes Highly Stretchable and Self-chargeable Supercapacitors", Mater. Horiz., 2020, vol. 7, pp. 2158-2167. 
Author's brief biography

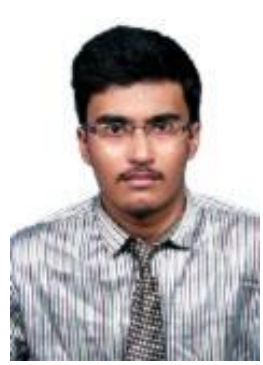

Karamjyoti Panigrahi: He was born at Nadia district of West Bengal, India in the year of 1992. He earned his B.Tech. in Electronics \& Instrumentation from West Bengal University of Technology (currently MAKAUT), Kolkata, West Bengal, India in 2013. He has earned his MTech \& PhD from School of

Materials Science \& Nanotechnology, Jadavpur University, Kolkata, WB, India in 2015 and 2020 respectively. Currently he has joined as Research Associate in Indian Institute of Science Education and Research (IISER), Pune-411108, India. During PhD tenure, his work encompasses investigation luminescent nanomaterials for energy efficient light generation, latent fingerprint development, anti-counterfeiting and solid-state flexible supercapacitor development. He is interested to explore the interdisciplinary domain of nanomaterials in transformer oil based nanofluids and electrocatalysis subsequently. He has awarded prestigious direct-SRF fellowship in Materials science domain from the Council of Scientific and Industrial Research (CSIR), India as well as InSc Research Excellence Award (2020). He also become a member of Electron microscopy Society of India (EMSI) in 2019 . 